\title{
On higher-power moments of $\Delta(x)$ (III)
}

\author{
by
}

\section{Wenguang Zhai (Jinan)}

1. Introduction and main results. Let $d(n)$ denote the Dirichlet divisor function and $\Delta(x)$ denote the error term of the sum $\sum_{n \leq x} d(n)$ for a large real variable $x$. Dirichlet proved that $\Delta(x)=O\left(x^{1 / 2}\right)$. The exponent $1 / 2$ was improved by many authors. The latest result reads

$$
\Delta(x) \ll x^{131 / 416}(\log x)^{26957 / 8320},
$$

proved by Huxley [3]. It is conjectured that

$$
\Delta(x)=O\left(x^{1 / 4+\varepsilon}\right),
$$

which is supported by the classical mean-square result

$$
\int_{1}^{T} \Delta^{2}(x) d x=\frac{(\zeta(3 / 2))^{4}}{6 \pi^{2} \zeta(3)} T^{3 / 2}+O\left(T \log ^{5} T\right)
$$

proved by Tong [10].

Tsang [11] studied the third- and fourth-power moments of $\Delta(x)$. He proved that

$$
\begin{aligned}
& \int_{2}^{T} \Delta^{3}(x) d x=\frac{3 c_{1}}{28 \pi^{3}} T^{7 / 4}+O\left(T^{7 / 4-\delta_{1}+\varepsilon}\right), \\
& \int_{2}^{T} \Delta^{4}(x) d x=\frac{3 c_{2}}{64 \pi^{4}} T^{2}+O\left(T^{2-\delta_{2}+\varepsilon}\right),
\end{aligned}
$$

where $\delta_{1}=1 / 14, \delta_{2}=1 / 23$, and

$$
c_{1}:=\sum_{\alpha, \beta, h \in \mathbb{N}}(\alpha \beta(\alpha+\beta))^{-3 / 2} h^{-9 / 4}|\mu(h)| d\left(\alpha^{2} h\right) d\left(\beta^{2} h\right) d\left((\alpha+\beta)^{2} h\right),
$$

2000 Mathematics Subject Classification: 11N37, 11M06.

Key words and phrases: power moment, Dirichlet divisor problem, Riemann zetafunction.

This work is supported by National Natural Science Foundation of China (Grant No. 10301018). 


$$
c_{2}:=\sum_{\substack{n, m, k, l \in \mathbb{N} \\ \sqrt{n}+\sqrt{m}=\sqrt{k}+\sqrt{l}}}(n m k l)^{-3 / 4} d(n) d(m) d(k) d(l) .
$$

Recently in [12] the author proved that (1.4) holds for $\delta_{1}=1 / 4$. In a forthcoming paper, Ivić and Sargos [7] proved that (1.4) holds for $\delta_{1}=7 / 20$. The author got this exponent independently. However, Professor Ivić kindly informed the author that the exponent $\delta_{1}=7 / 20$ had already been obtained by Professor Tsang several years ago but he had never published this result.

Following Tsang's approach, in [12] the author proved that (1.5) holds for $\delta_{2}=2 / 41$. This approach used the method of exponential sums. In particular, if the exponent pair conjecture is true, namely, if $(\varepsilon, 1 / 2+\varepsilon)$ is an exponent pair, then (1.5) holds for $\delta_{2}=1 / 14$. However, in [7] Ivić and Sargos ingeniously proved a substantially better result. They proved that (1.5) holds for $\delta_{2}=1 / 12$.

In this paper, combining the method of [7] and a recent deep result of Robert and Sargos [9], we shall prove the following

THEOREM 1. We have

$$
\int_{2}^{T} \Delta^{4}(x) d x=\frac{3 c_{2}}{64 \pi^{4}} T^{2}+O\left(T^{53 / 28+\varepsilon}\right) .
$$

The theorem is also true for other error terms. Let $P(x)$ denote the error term of the Gauss circle problem, which is an error term similar to $\Delta(x)$. Let $a(n)$ be the Fourier coefficients of a holomorphic cusp form of weight $\kappa=2 n \geq 12$ for the full modular group and define

$$
A(x):=\sum_{n \leq x}^{\prime} a(n), \quad x \geq 2 .
$$

We then have the following two corollaries, which improve the previous results $([2],[11],[12])$.

Corollary 1. We have

$$
\int_{2}^{T} P^{4}(x) d x=C T^{2}+O\left(T^{53 / 28+\varepsilon}\right)
$$

Corollary 2. We have

$$
\int_{1}^{T} A^{4}(x) d x=B_{\kappa} T^{2 \kappa}+O\left(T^{2 \kappa-3 / 28+\varepsilon}\right) .
$$

Now consider $E(t)$, defined by

$$
E(t):=\int_{0}^{t}|\zeta(1 / 2+i u)|^{2} d u-t \log (t / 2 \pi)-(2 \gamma-1) t, \quad t \geq 2
$$


Tsang [11] also studied the fourth-power moment of $E(t)$ by using Atkinson's formula [1] and proved that

$$
\int_{2}^{T} E^{4}(t) d t=\frac{3}{8 \pi} c_{2} T^{2}+O\left(T^{2-\delta_{3}+\varepsilon}\right)
$$

with some unspecified constant $\delta_{3}>0$.

Ivić [4] used a different way to study the higher power moments of $E(t)$. His approach is as follows. Let

$$
\Delta^{*}(x):=\frac{1}{2} \sum_{n \leq 4 x}(-1)^{n} d(n)-x(\log x+2 \gamma-1), \quad x \geq 1 .
$$

Then for $1 \ll N \ll x$, we have $[6]$

$$
\begin{aligned}
\Delta^{*}(x)=\frac{1}{\pi \sqrt{2}} \sum_{n \leq N}(-1)^{n} d(n) n^{-3 / 4} x^{1 / 4} \cos (4 & \pi \sqrt{n x}-\pi / 4) \\
& +O\left(x^{1 / 2+\varepsilon} N^{-1 / 2}\right) .
\end{aligned}
$$

Jutila [8] proved that

$$
\int_{0}^{T}\left(E(t)-2 \pi \Delta^{*}\left(\frac{t}{2 \pi}\right)\right)^{2} d t \ll T^{4 / 3} \log ^{3} T,
$$

which means that $E(t)$ is well approximated by $2 \pi \Delta^{*}(t / 2 \pi)$ at least in the mean square sense. From (1.13) Ivić [4] deduced that

$$
\int_{0}^{T} E^{4}(t) d t=(2 \pi)^{5} \int_{0}^{T / 2 \pi}\left(\Delta^{*}(t)\right)^{4} d t+O\left(T^{23 / 12} \log ^{3 / 2} T\right) .
$$

Thus the fourth-power moment of $E(t)$ was transformed into the fourthpower moment of $\Delta^{*}(t)$, which can be dealt with in the same way as the fourth-power moment of $\Delta(x)$. By Tsang's result [11], Ivić deduced from (1.14) that (1.10) holds for $\delta_{3}=1 / 23$. In [7], Ivić and Sargos proved that one can take $\delta_{3}=1 / 12$.

It is easy to see that $1 / 12$ is the limit of this approach since it is the limit of Jutila's result (1.13). In this paper, we shall use a different way to prove the following

THEOREM 2. We have

$$
\int_{2}^{T} E^{4}(t) d t=\frac{3}{8 \pi} c_{2} T^{2}+O\left(T^{53 / 28+\varepsilon}\right) .
$$

REMARK. The proof of Theorem 2 does not use (1.13) and it is actually a generalization of the approach used in the author's paper [13]. In [14] the author used a similar method to study the third-power moment of $E(t)$. 
Acknowledgements. I am very grateful to the referee for many valuable suggestions. In particular, he gave a direct proof of Lemma 3, which is much easier than my previous one. I wish to thank Professor Ivić and Professor Robert, who kindly sent me the papers [7] and [9], respectively.

Notations. Throughout this paper, $[x]$ denotes the integer part of $x$, $\|x\|$ denotes the distance from $x$ to the integer nearest to $x, n \sim N$ means $N<n \leq 2 N, n \asymp N$ means $C_{1} N<n \leq C_{2} N$ for positive constants $C_{1}<C_{2}$, and $\varepsilon$ always denotes a small positive constant which may be different at different places. We shall use the estimate $d(n) \ll n^{\varepsilon}$ freely.

2. The spacing problem of the square roots. In the proofs of Theorems 1 and 2, the sums and differences of square roots will appear in the exponential. Thus we should study the spacing problem of the square roots.

We need the following lemmas. Lemma 1 is a special case of a new result proved in Robert and Sargos [9], which also plays an important role in this paper. Lemma 2 is Lemma 3 of Tsang [11]. Lemma 3 provides an upper bound for the number of solutions of the inequality

$$
\left|n_{1}^{1 / 2}+n_{2}^{1 / 2} \pm n_{3}^{1 / 2}-n_{4}^{1 / 2}\right|<\Delta, \quad n_{j} \sim N_{j} \quad(j=1,2,3,4),
$$

where $N_{j} \geq 2(j=1,2,3,4)$ are real numbers. Lemma 4 is essentially Lemma 3 of Ivić and Sargos [7], but we added the case $\alpha \ll 1$. Lemma 5 is essentially Lemma 5 of [7], but the term $K \min \left(M, M^{\prime}, L\right)$ therein is superfluous since we add the condition $|\sqrt{n}+\sqrt{m}-\sqrt{k}-\sqrt{l}|>0$ in Lemma 5, and so we give a new proof here. Lemma 6 is Lemma 6 of [7].

Lemma 1. Suppose $N \geq 2, \Delta>0$. Let $\mathcal{A}(N ; \Delta)$ denote the number of solutions of the inequality

Then

$$
\left|n_{1}^{1 / 2}+n_{2}^{1 / 2}-n_{3}^{1 / 2}-n_{4}^{1 / 2}\right|<\Delta, \quad n_{j} \sim N \quad(j=1,2,3,4) .
$$

$$
\mathcal{A}(N ; \Delta) \ll\left(\Delta N^{7 / 2}+N^{2}\right) N^{\varepsilon} .
$$

Lemma 2. If $n, m, k, l \in \mathbb{N}$ are such that $\sqrt{n}+\sqrt{m} \pm \sqrt{k}-\sqrt{l} \neq 0$, then respectively,

$$
|\sqrt{n}+\sqrt{m} \pm \sqrt{k}-\sqrt{l}| \gg \max (n, m, k, l)^{-7 / 2} .
$$

Lemma 3. Suppose $N_{j} \geq 2(j=1,2,3,4), \Delta>0$. Let $\mathcal{A}_{ \pm}\left(N_{1}, N_{2}, N_{3}\right.$, $\left.N_{4} ; \Delta\right)$ denote the number of solutions of inequality (2.1). Then

$$
\mathcal{A}_{ \pm}\left(N_{1}, N_{2}, N_{3}, N_{4} ; \Delta\right) \ll \prod_{j=1}^{4}\left(\Delta^{1 / 4} N_{j}^{7 / 8}+N_{j}^{1 / 2}\right) N_{j}^{\varepsilon} .
$$

Proof. We use a combinatorial argument. Let $\left\{a_{i}\right\}$ and $\left\{b_{i}\right\}$ be two finite sequences of real numbers. Let $\Delta>0$. Suppose $u_{0}$ and $J$ (a positive integer) 
are chosen so that $\left\{a_{i}\right\} \subset\left(u_{0}, u_{0}+J \Delta\right],\left\{b_{i}\right\} \subset\left(u_{0}, u_{0}+J \Delta\right]$. Divide this interval into the abutting subintervals $I_{j}:=\left(u_{0}+j \Delta, u_{0}+(j+1) \Delta\right]$ for $j=0,1, \ldots, J-1$ and then let

$$
N_{j}(A):=\#\left\{i: a_{i} \in I_{j}\right\}, \quad N_{j}(B):=\#\left\{i: b_{i} \in I_{j}\right\} .
$$

If $\left|a_{r}-b_{s}\right| \leq \Delta$, then either both $a_{r}$ and $b_{s}$ lie in the same subinterval $I_{j}$, or they lie in adjacent subintervals $I_{j}$ and $I_{j+1}$. Hence

$$
\begin{aligned}
\#\{(r, s) & \left.:\left|a_{r}-b_{s}\right| \leq \Delta\right\} \\
& \leq \sum_{j} N_{j}(A) N_{j}(B)+\sum_{j} N_{j}(A) N_{j+1}(B)+\sum_{j} N_{j+1}(A) N_{j}(B) \\
& \leq 3\left(\sum_{j} N_{j}(A)^{2}\right)^{1 / 2}\left(\sum_{j} N_{j}(B)^{2}\right)^{1 / 2}
\end{aligned}
$$

by Cauchy-Schwarz's inequality. On the other hand, we have

$$
\sum_{j} N_{j}(A)^{2}=\sum_{j} \#\left\{\left(r, r^{\prime}\right): a_{r}, a_{r^{\prime}} \in I_{j}\right\} \leq \#\left\{\left(r, r^{\prime}\right):\left|a_{r}-a_{r^{\prime}}\right| \leq \Delta\right\},
$$

and similarly for $\sum_{j} N_{j}(B)^{2}$. Thus

$$
\begin{aligned}
& \#\left\{(r, s):\left|a_{r}-b_{s}\right| \leq \Delta\right\} \\
& \quad \leq 3\left(\#\left\{\left(r, r^{\prime}\right):\left|a_{r}-a_{r^{\prime}}\right| \leq \Delta\right\}\right)^{1 / 2}\left(\#\left\{\left(s, s^{\prime}\right):\left|b_{s}-b_{s^{\prime}}\right| \leq \Delta\right\}\right)^{1 / 2} .
\end{aligned}
$$

Suppose $n_{j}, n_{j}^{\prime} \sim N_{j}(j=1,2,3,4)$. Applying (2.2) to the sequences $A=\left\{\sqrt{n_{1}}+\sqrt{n_{2}}\right\}$ and $B=\left\{\sqrt{n_{3}}+\sqrt{n_{4}}\right\}$, we get

$$
\begin{aligned}
\mathcal{A}_{-} & \left(N_{1}, N_{2}, N_{3}, N_{4}\right) \\
= & \#\left\{\left(n_{1}, n_{2}, n_{3}, n_{4}\right):\left|n_{1}^{1 / 2}+n_{2}^{1 / 2}-n_{3}^{1 / 2}-n_{4}^{1 / 2}\right| \leq \Delta\right\} \\
\leq & 3\left(\#\left\{\left(n_{1}, n_{2}, n_{1}^{\prime}, n_{2}^{\prime}\right):\left|n_{1}^{1 / 2}+n_{2}^{1 / 2}-n_{1}^{\prime 1 / 2}-n_{2}^{\prime 1 / 2}\right| \leq \Delta\right\}\right)^{1 / 2} \\
& \times\left(\#\left\{\left(n_{3}, n_{4}, n_{3}^{\prime}, n_{4}^{\prime}\right):\left|n_{3}^{1 / 2}+n_{4}^{1 / 2}-n_{3}^{\prime 1 / 2}-n_{4}^{\prime 1 / 2}\right| \leq \Delta\right\}\right)^{1 / 2} .
\end{aligned}
$$

Applying the previous bound to the sequences $A_{1}=\left\{n_{1}^{1 / 2}-n_{1}^{\prime 1 / 2}\right\}, B_{1}=$ $\left\{n_{2}^{1 / 2}-n_{2}^{\prime 1 / 2}\right\}$, and $A_{2}=\left\{n_{3}^{1 / 2}-n_{3}^{1 / 2}\right\}, B_{2}=\left\{n_{4}^{1 / 2}-n_{4}^{\prime 1 / 2}\right\}$, respectively, we get

$$
\mathcal{A}_{-}\left(N_{1}, N_{2}, N_{3}, N_{4}\right) \leq 9 \prod_{j=1}^{4} \mathcal{A}_{-}\left(N_{j}, N_{j}, N_{j}, N_{j}\right)^{1 / 4},
$$

which combined with Lemma 1 gives Lemma 3 for the "-" case. The proof for the "+" case is similar.

Lemma 4. Suppose $K \geq 10, \alpha, \beta \in \mathbb{R}, 2 K^{-1 / 2} \leq|\alpha| \ll K^{1 / 2}$ and $0<\delta<1 / 2$. Then

$$
\#\{k \sim K:\|\beta+\alpha \sqrt{k}\|<\delta\} \ll K \delta+K^{1 / 2+\varepsilon} .
$$


Proof. Without loss of generality, suppose $\alpha>0$. Let $\mathcal{N}=\#\{k \sim K$ : $\|\beta+\alpha \sqrt{k}\|<\delta\}$. If $1 \ll \alpha \ll K^{1 / 2}$, from Lemma 3 of Ivić and Sargos [7] we get

$$
\mathcal{N} \ll K \delta+|\alpha|^{1 / 2} K^{1 / 4+\varepsilon}+K^{1 / 2+\varepsilon} \ll K \delta+K^{1 / 2+\varepsilon} .
$$

Now suppose $2 K^{-1 / 2} \leq \alpha \ll 1$. Since $\|t\|$ is a periodic function with period 1 , we suppose $0<\beta \leq 1$. If $\|\beta+\alpha \sqrt{k}\|<\delta$, then there exists a unique $l \in[\alpha \sqrt{K}, 2 \alpha \sqrt{K}+2]$ such that

$$
(l-\beta-\delta)^{2} / \alpha^{2}<k \leq(l-\beta+\delta)^{2} / \alpha^{2},
$$

which implies

$$
\begin{aligned}
\mathcal{N} & \ll \sum_{l \sim \alpha \sqrt{K}}\left(\left[(l-\beta+\delta)^{2} / \alpha^{2}\right]-\left[(l-\beta-\delta)^{2} / \alpha^{2}\right]\right) \\
& \ll \sum_{l \sim \alpha \sqrt{K}}\left((l-\beta+\delta)^{2} / \alpha^{2}-(l-\beta-\delta)^{2} / \alpha^{2}+1\right) \\
& \ll K \delta+K^{1 / 2}
\end{aligned}
$$

if we notice $\alpha \ll 1$.

Lemma 5. Suppose $1 \leq N \leq M, 1 \leq L \leq K, N \leq L, M \asymp K, 0<$ $\Delta \ll K^{1 / 2}$. Let $\mathcal{A}_{1}(N, M, K, L ; \Delta)$ denote the number of solutions of the inequality

$$
0<|\sqrt{n}+\sqrt{m}-\sqrt{k}-\sqrt{l}|<\Delta
$$

with $n \sim N, m \sim M, k \sim K, l \sim L$. Then

$$
\mathcal{A}_{1}(N, M, K, L ; \Delta) \ll \Delta K^{1 / 2} N M L+N L K^{1 / 2+\varepsilon} .
$$

In particular, if $\Delta K^{1 / 2} \gg 1$, then

$$
\mathcal{A}_{1}(N, M, K, L ; \Delta) \ll \Delta K^{1 / 2} N M L .
$$

Proof. If $(n, m, k, l)$ satisfies $|\sqrt{n}+\sqrt{m}-\sqrt{k}-\sqrt{l}|<\Delta$, then

$$
m=k+2 k^{1 / 2}(\sqrt{l}-\sqrt{n})+(\sqrt{l}-\sqrt{n})^{2}+u
$$

with $|u| \leq C \Delta K^{1 / 2}$ for some absolute constant $C>0$. Hence the quantity $\mathcal{A}_{1}(N, M, K, L ; \Delta)$ does not exceed the number of solutions of the inequality

$$
\left|2 k^{1 / 2}(\sqrt{l}-\sqrt{n})+(\sqrt{l}-\sqrt{n})^{2}+k-m\right|<C \Delta K^{1 / 2}
$$

with $n \sim N, m \sim M, k \sim K, l \sim L$.

If $\Delta K^{1 / 2} \gg 1$, then for fixed $(n, k, l)$, the number of $m$ for which $(2.5)$ holds is $\ll 1+\Delta K^{1 / 2} \ll \Delta K^{1 / 2}$ if we notice $K \asymp M$. Hence

$$
\mathcal{A}_{1}(N, M, K, L ; \Delta) \ll \Delta K^{1 / 2} N M L .
$$

Now suppose $\Delta K^{1 / 2} \leq 1 / 4 C$. For fixed $(n, k, l)$, there is at most one $m$ such that (2.5) holds. If such an $m$ exists, then we have

$$
\left\|2 k^{1 / 2}(\sqrt{l}-\sqrt{n})+(\sqrt{l}-\sqrt{n})^{2}\right\|<C \Delta K^{1 / 2} .
$$


We shall use Lemma 4 to bound the number of solutions of (2.6) with $\alpha=2(\sqrt{l}-\sqrt{n}), \beta=(\sqrt{l}-\sqrt{n})^{2}$. Let $\mathcal{C}_{1}$ denote the number of solutions of (2.6) with $|\alpha| \geq 2 K^{-1 / 2}$, and $\mathcal{C}_{2}$ the number of solutions with $|\alpha|<2 K^{-1 / 2}$. By Lemma 4 we get

$$
\mathcal{C}_{1} \ll \Delta K^{1 / 2} N M L+N L K^{1 / 2+\varepsilon}
$$

if we notice $M \asymp K$. Now we estimate $\mathcal{C}_{2}$. From $|\alpha|<2 K^{-1 / 2}$, we get $N \asymp L$. If $l=n$, from $(2.5)$ we get $k=m$. This contradicts $|\sqrt{n}+\sqrt{m}-\sqrt{k}-\sqrt{l}|>0$. Thus $l \neq n$. From

$$
2 K^{-1 / 2}>|\sqrt{l}-\sqrt{n}|=\frac{|l-n|}{\sqrt{l}+\sqrt{n}} \geq \frac{1}{\sqrt{l}+\sqrt{n}} \geq 1 / 2 \sqrt{2 L}
$$

we get $L \gg K$ and thus $N \asymp M \asymp K \asymp L$. So we have

$$
\mathcal{C}_{2} \ll \#\left\{(l, n):|\alpha|<2 K^{-1 / 2}\right\} \times \#\{k\} \ll K^{2},
$$

which can be absorbed into the estimate of $\mathcal{C}_{1}$. This completes the proof of Lemma 5.

Lemma 6. Suppose $1 \leq N \leq M \leq K \asymp L, 0<\Delta \ll L^{1 / 2}$. Let $\mathcal{A}_{2}(N, M, K, L ; \Delta)$ denote the number of solutions of the inequality

$$
|\sqrt{n}+\sqrt{m}+\sqrt{k}-\sqrt{l}|<\Delta
$$

with $n \sim N, m \sim M, k \sim K, l \sim L$. Then

$$
\mathcal{A}_{2}(N, M, K, L ; \Delta) \ll \Delta L^{1 / 2} N M K+N M K^{1 / 2+\varepsilon} .
$$

In particular, if $\Delta L^{1 / 2} \gg 1$, then

$$
\mathcal{A}_{2}(N, M, K, L ; \Delta) \ll \Delta L^{1 / 2} N M K .
$$

3. Proof of Theorem 1. Suppose $T \geq 10$. It suffices to evaluate the integral $\int_{T}^{2 T} \Delta^{4}(x) d x$. Suppose $y=T^{3 / 4}$. For any $T \leq x \leq 2 T$, by the truncated Voronoï formula, we get

$$
\Delta(x)=\frac{1}{\sqrt{2} \pi} \mathcal{R}+O\left(x^{1 / 2+\varepsilon} y^{-1 / 2}\right)
$$

where

$$
\mathcal{R}:=\mathcal{R}(x)=x^{1 / 4} \sum_{n \leq y} \frac{d(n)}{n^{3 / 4}} \cos \left(4 \pi \sqrt{x n}-\frac{\pi}{4}\right) .
$$

We have

$$
\begin{aligned}
\int_{T}^{2 T} \Delta^{4}(x) d x & =\frac{1}{4 \pi^{4}} \int_{T}^{2 T} \mathcal{R}^{4} d x+O\left(T^{9 / 4+\varepsilon} y^{-1 / 2}+T^{3+\varepsilon} y^{-2}\right) \\
& =\frac{1}{4 \pi^{4}} \int_{T}^{2 T} \mathcal{R}^{4} d x+O\left(T^{15 / 8+\varepsilon}\right)
\end{aligned}
$$


Let

$g=g(n, m, k, l):=(n m k l)^{-3 / 4} d(n) d(m) d(k) d(l) \quad$ for $n, m, k, l \leq y$, and $g=0$ otherwise.

Equation (3.4) of Tsang [11] reads

$$
\mathcal{R}^{4}=S_{1}(x)+S_{2}(x)+S_{3}(x)+S_{4}(x),
$$

where

$$
\begin{aligned}
& S_{1}(x):=\frac{3}{8} \sum_{\sqrt{n}+\sqrt{m}=\sqrt{k}+\sqrt{l}} g x, \\
& S_{2}(x):=\frac{3}{8} \sum_{\sqrt{n}+\sqrt{m} \neq \sqrt{k}+\sqrt{l}} g x \cos (4 \pi(\sqrt{n}+\sqrt{m}-\sqrt{k}-\sqrt{l}) \sqrt{x}), \\
& S_{3}(x):=\frac{1}{2} \sum_{g} g x \sin (4 \pi(\sqrt{n}+\sqrt{m}+\sqrt{k}-\sqrt{l}) \sqrt{x}), \\
& S_{4}(x):=-\frac{1}{8} \sum g x \cos (4 \pi(\sqrt{n}+\sqrt{m}+\sqrt{k}+\sqrt{l}) \sqrt{x}) .
\end{aligned}
$$

From (3.7) of [11] we get

$$
\int_{T}^{2 T} S_{1}(x) d x=\frac{3 c_{2}}{8} \int_{T}^{2 T} x d x+O\left(T^{2-3 / 16+\varepsilon}\right) .
$$

From the first derivative test we get

$$
\int_{T}^{2 T} S_{4}(x) d x \ll T^{3 / 2+\varepsilon} y^{1 / 2} \ll T^{15 / 8+\varepsilon} .
$$

Now let us consider the contribution of $S_{2}(x)$. By the first derivative test we get

$$
\begin{aligned}
\int_{T}^{2 T} S_{2}(x) d x & \ll \sum_{\substack{n, m, k, l \leq y \\
\sqrt{n}+\sqrt{m} \neq \sqrt{k}+\sqrt{l}}} g \min \left(T^{2}, \frac{T^{3 / 2}}{|\sqrt{n}+\sqrt{m}-\sqrt{k}-\sqrt{l}|}\right) \\
& \ll T^{\varepsilon} G(N, M, K, L),
\end{aligned}
$$

where

$$
\begin{gathered}
G(N, M, K, L)=\sum_{1} g \min \left(T^{2}, \frac{T^{3 / 2}}{|\sqrt{n}+\sqrt{m}-\sqrt{k}-\sqrt{l}|}\right), \\
\mathrm{SC}\left(\sum_{1}\right): \sqrt{n}+\sqrt{m} \neq \sqrt{k}+\sqrt{l}, 1 \leq N \leq M \leq y, 1 \leq L \leq K \leq y, \\
N \leq L, n \sim N, m \sim M, k \sim K, l \sim L .
\end{gathered}
$$

If $M \geq 100 K$, then $|\sqrt{n}+\sqrt{m}-\sqrt{k}-\sqrt{l}| \gg M^{1 / 2}$, so the trivial estimate 
yields

$$
G(N, M, K, L) \ll \frac{T^{3 / 2+\varepsilon} N M K L}{(N M K L)^{3 / 4} M^{1 / 2}} \ll T^{3 / 2+\varepsilon} y^{1 / 2} \ll T^{15 / 8+\varepsilon} .
$$

If $K>100 M$, we get the same estimate. So later we always suppose that $M \asymp K$.

Let $\eta=\sqrt{n}+\sqrt{m}-\sqrt{k}-\sqrt{l}$. Write

$$
G(N, M, K, L, R)=G_{1}+G_{2}+G_{3},
$$

where

$$
\begin{aligned}
G_{1} & :=T^{2} \sum_{|\eta| \leq T^{-1 / 2}} g, \\
G_{2} & :=T^{3 / 2} \sum_{T^{-1 / 2}<|\eta| \leq 1} g|\eta|^{-1}, \\
G_{3} & :=T^{3 / 2} \sum_{|\eta| \gg 1} g|\eta|^{-1} .
\end{aligned}
$$

We estimate $G_{1}$ first. From $|\eta| \leq T^{-1 / 2}$ we get $M \asymp K \gg T^{1 / 7}$ via Lemma 2 . By Lemma 5 we get

$$
\begin{aligned}
G_{1} & \ll \frac{T^{2+\varepsilon}}{(N M K L)^{3 / 4}} \mathcal{A}_{1}\left(N, M, K, L ; T^{-1 / 2}\right) \\
& \ll \frac{T^{2+\varepsilon}}{(N M K L)^{3 / 4}}\left(T^{-1 / 2} K^{1 / 2} N M L+N L K^{1 / 2}\right) \\
& \ll T^{3 / 2+\varepsilon}(N L)^{1 / 4}+T^{2+\varepsilon}(N L)^{1 / 4} K^{-1} \\
& \ll T^{3 / 2+\varepsilon} y^{1 / 2}+T^{2+\varepsilon}(N L)^{1 / 4} K^{-1} \\
& \ll T^{15 / 8+\varepsilon}+T^{2+\varepsilon}(N L)^{1 / 4} K^{-1} .
\end{aligned}
$$

By Lemma 3 we get (notice $N \leq L \leq K$ )

$$
\begin{aligned}
G_{1} \ll & \frac{T^{2+\varepsilon}}{(N M K L)^{3 / 4}} \mathcal{A}_{-}\left(N, M, K, L ; T^{-1 / 2}\right) \\
\ll & \frac{T^{2+\varepsilon}}{(N M K L)^{3 / 4}}\left(T^{-1 / 8} N^{7 / 8}+N^{1 / 2}\right)\left(T^{-1 / 8} L^{7 / 8}+L^{1 / 2}\right) \\
& \quad \times\left(T^{-1 / 4} K^{7 / 4}+K\right) \\
\ll & T^{2+\varepsilon}\left(T^{-1 / 8} N^{1 / 8}+N^{-1 / 4}\right)\left(T^{-1 / 8} L^{1 / 8}+L^{-1 / 4}\right) \\
& \times\left(T^{-1 / 4} K^{1 / 4}+K^{-1 / 2}\right) \\
\ll & T^{2+\varepsilon}\left(T^{-1 / 4}(N L)^{1 / 8}+T^{-1 / 8} L^{1 / 8} N^{-1 / 4}+(N L)^{-1 / 4}\right) \\
& \times\left(T^{-1 / 4} K^{1 / 4}+K^{-1 / 2}\right)
\end{aligned}
$$




$$
\begin{aligned}
\ll & T^{2+\varepsilon} T^{-1 / 4}(N L)^{1 / 8}\left(T^{-1 / 4} K^{1 / 4}+K^{-1 / 2}\right) \\
& +T^{2+\varepsilon}\left(T^{-1 / 8} L^{3 / 8}(N L)^{-1 / 4}+(N L)^{-1 / 4}\right)\left(T^{-1 / 4} K^{1 / 4}+K^{-1 / 2}\right) \\
\ll & T^{3 / 2+\varepsilon} y^{1 / 2}+T^{7 / 4+\varepsilon} K^{-1 / 4} \\
& \quad+T^{2+\varepsilon}\left(T^{-1 / 4} K^{1 / 4}+K^{-1 / 2}\right)\left(T^{-1 / 8} K^{3 / 8}+1\right)(N L)^{-1 / 4} \\
& \ll T^{15 / 8+\varepsilon}+T^{2+\varepsilon} K^{-1 / 2}\left(T^{-1 / 4} K^{3 / 4}+1\right)\left(T^{-1 / 8} K^{3 / 8}+1\right)(N L)^{-1 / 4} \\
\ll & T^{15 / 8+\varepsilon}+T^{2+\varepsilon} K^{-1 / 2}\left(T^{-3 / 8} K^{9 / 8}+1\right)(N L)^{-1 / 4} .
\end{aligned}
$$

From (3.8) and (3.9) we get

$$
\begin{aligned}
G_{1} \ll & T^{15 / 8+\varepsilon} \\
& +T^{2+\varepsilon} \min \left((N L)^{1 / 4} K^{-1}, K^{-1 / 2}\left(T^{-3 / 8} K^{9 / 8}+1\right)(N L)^{-1 / 4}\right) \\
\ll & T^{15 / 8+\varepsilon} \\
& +T^{2+\varepsilon}\left((N L)^{1 / 4} K^{-1}\right)^{1 / 2}\left(K^{-1 / 2}\left(T^{-3 / 8} K^{9 / 8}+1\right)(N L)^{-1 / 4}\right)^{1 / 2} \\
& \ll T^{15 / 8+\varepsilon}+T^{2+\varepsilon} K^{-3 / 4}\left(T^{-3 / 16} K^{9 / 16}+1\right) \\
\ll & T^{15 / 8+\varepsilon}+T^{2+\varepsilon} K^{-3 / 4} \ll T^{53 / 28+\varepsilon}
\end{aligned}
$$

if we notice $K \gg T^{1 / 7}$.

Now we estimate $G_{2}$. By a splitting argument we get the estimate

$$
G_{2} \ll \frac{T^{3 / 2+\varepsilon}}{(N M K L)^{3 / 4} \delta} \sum_{\substack{\delta<|\eta| \leq 2 \delta \\ \eta \neq 0}} 1
$$

for some $T^{-1 / 2} \leq \delta \leq 1$. By Lemma 5 we get

$$
\begin{aligned}
G_{2} & \ll \frac{T^{3 / 2+\varepsilon}}{(N M K L)^{3 / 4} \delta} \mathcal{A}_{1}(N, M, K, L ; 2 \delta) \\
& \ll \frac{T^{3 / 2+\varepsilon}}{(N M K L)^{3 / 4} \delta}\left(\delta K^{1 / 2} N M L+N L K^{1 / 2}\right) \\
& \ll T^{3 / 2+\varepsilon} y^{1 / 2}+T^{3 / 2+\varepsilon}(K \delta)^{-1}(N L)^{1 / 4} \\
& \ll T^{15 / 8+\varepsilon}+T^{3 / 2+\varepsilon}(K \delta)^{-1}(N L)^{1 / 4} .
\end{aligned}
$$

By Lemma 3 we get (notice $N \leq L \leq K$ )

$$
\begin{aligned}
G_{2} \ll & \frac{T^{3 / 2+\varepsilon}}{(N M K L)^{3 / 4} \delta} \mathcal{A}_{-}(N, M, K, L ; 2 \delta) \\
\ll & \frac{T^{3 / 2+\varepsilon}}{(N M K L)^{3 / 4} \delta}\left(\delta^{1 / 4} N^{7 / 8}+N^{1 / 2}\right)\left(\delta^{1 / 4} L^{7 / 8}+L^{1 / 2}\right) \\
& \times\left(\delta^{1 / 2} K^{7 / 4}+K\right)
\end{aligned}
$$




$$
\begin{aligned}
\ll & T^{3 / 2+\varepsilon}\left(N^{1 / 8}+N^{-1 / 4} \delta^{-1 / 4}\right)\left(L^{1 / 8}+L^{-1 / 4} \delta^{-1 / 4}\right) \\
& \times\left(K^{1 / 4}+K^{-1 / 2} \delta^{-1 / 2}\right) \\
\ll & T^{3 / 2+\varepsilon}\left((N L)^{1 / 8}+L^{1 / 8} N^{-1 / 4} \delta^{-1 / 4}+(N L)^{-1 / 4} \delta^{-1 / 2}\right) \\
& \times\left(K^{1 / 4}+K^{-1 / 2} \delta^{-1 / 2}\right) \\
\ll & T^{3 / 2+\varepsilon}(N L)^{1 / 8} K^{1 / 4}+T^{3 / 2+\varepsilon}(N L)^{1 / 8} K^{-1 / 2} \delta^{-1 / 2} \\
& +T^{3 / 2+\varepsilon}\left(K^{1 / 4}+K^{-1 / 2} \delta^{-1 / 2}\right)\left(L^{3 / 8} \delta^{1 / 4}+1\right)(N L)^{-1 / 4} \delta^{-1 / 2} \\
\ll & T^{3 / 2+\varepsilon} y^{1 / 2}+T^{3 / 2+\varepsilon} \delta^{-1 / 2} \\
& +T^{3 / 2+\varepsilon} K^{-1 / 2} \delta^{-1}\left(K^{3 / 4} \delta^{1 / 2}+1\right)\left(K^{3 / 8} \delta^{1 / 4}+1\right)(N L)^{-1 / 4} \\
\ll & T^{15 / 8+\varepsilon}+T^{3 / 2+\varepsilon} K^{-1 / 2} \delta^{-1}\left(K^{9 / 8} \delta^{3 / 4}+1\right)(N L)^{-1 / 4},
\end{aligned}
$$

where the bound $\delta \gg T^{-1 / 2}$ was applied to the term $T^{3 / 2+\varepsilon} \delta^{-1 / 2}$.

From (3.12) and (3.13) we get

$$
\begin{aligned}
G_{2} & \ll T^{15 / 8+\varepsilon}+\frac{T^{3 / 2+\varepsilon}}{\delta} \min \left(\frac{(N L)^{1 / 4}}{K}, \frac{K^{9 / 8} \delta^{3 / 4}+1}{K^{1 / 2}(N L)^{1 / 4}}\right) \\
& \ll T^{15 / 8+\varepsilon}+\frac{T^{3 / 2+\varepsilon}}{\delta}\left(\frac{(N L)^{1 / 4}}{K}\right)^{1 / 2}\left(\frac{K^{9 / 8} \delta^{3 / 4}+1}{K^{1 / 2}(N L)^{1 / 4}}\right)^{1 / 2} \\
& \ll T^{15 / 8+\varepsilon}+T^{3 / 2+\varepsilon} \delta^{-1} K^{-3 / 4}\left(K^{9 / 16} \delta^{3 / 8}+1\right) .
\end{aligned}
$$

If $\delta \gg K^{-3 / 2}$, then (3.14) implies (recall $\delta \gg T^{-1 / 2}$ )

$$
G_{2} \ll T^{15 / 8+\varepsilon}+T^{3 / 2+\varepsilon} K^{-3 / 16} \delta^{-5 / 8} \ll T^{15 / 8+\varepsilon} .
$$

If $\delta \ll K^{-3 / 2}$, then (3.14) becomes

$$
G_{2} \ll T^{15 / 8+\varepsilon}+T^{3 / 2+\varepsilon} \delta^{-1} K^{-3 / 4} .
$$

Since $\delta \gg K^{-7 / 2}$ by Lemma 2 and $\delta \gg T^{-1 / 2}$, we get

$$
\delta^{-1} \ll \min \left(K^{7 / 2}, T^{1 / 2}\right)
$$

and thus from (3.16) we get

$$
\begin{aligned}
G_{2} & \ll T^{15 / 8+\varepsilon}+\min \left(T^{2+\varepsilon} K^{-3 / 4}, T^{3 / 2+\varepsilon} K^{11 / 4}\right) \\
& \ll T^{15 / 8+\varepsilon}+\left(T^{2+\varepsilon} K^{-3 / 4}\right)^{11 / 14}\left(T^{3 / 2+\varepsilon} K^{11 / 4}\right)^{3 / 14} \\
& \ll T^{53 / 28+\varepsilon} .
\end{aligned}
$$

For $G_{3}$, by a splitting argument and Lemma 5 again (notice $|\eta| \gg 1$ ) we get 


$$
\begin{aligned}
G_{3} & \ll \frac{T^{3 / 2+\varepsilon}}{(N M K L)^{3 / 4} \delta} \sum_{\delta<|\eta| \leq 2 \delta, \delta \gg 1} 1 \\
& \ll \frac{T^{3 / 2+\varepsilon}}{(N M K L)^{3 / 4}} K^{1 / 2} N M L \ll T^{3 / 2+\varepsilon} y^{1 / 2} \ll T^{15 / 8+\varepsilon} .
\end{aligned}
$$

Combining (3.6), (3.7), (3.10) and (3.15)-(3.18) we get

$$
\int_{T}^{2 T} S_{2}(x) d x \ll T^{53 / 28+\varepsilon} .
$$

In the same way, by Lemmas 3 and 6 , we can show that

$$
\int_{T}^{2 T} S_{3}(x) d x \ll T^{53 / 28+\varepsilon} .
$$

From (3.2)-(3.5), (3.19) and (3.20) we get

$$
\int_{T}^{2 T} \Delta^{4}(x) d x=\frac{3 c_{2}}{32 \pi^{4}} \int_{T}^{2 T} x d x+O\left(T^{53 / 28+\varepsilon}\right),
$$

which implies Theorem 1 immediately.

4. Preliminary lemmas for Theorem 2. In order to prove Theorem 2 , we need the following lemmas.

LEMMA 7. We have

$$
E(t)=\Sigma_{1}(t)+\Sigma_{2}(t)+O\left(\log ^{2} t\right)
$$

with

$$
\begin{aligned}
& \Sigma_{1}(t):=\frac{1}{\sqrt{2}} \sum_{n \leq N} h(t, n) \cos (f(t, n)), \\
& \Sigma_{2}(t):=-2 \sum_{n \leq N^{\prime}} d(n) n^{-1 / 2}\left(\log \frac{t}{2 \pi n}\right)^{-1} \cos \left(t \log \frac{t}{2 \pi n}-t+\frac{\pi}{4}\right), \\
& h(t, n):=(-1)^{n} d(n) n^{-1 / 2}\left(\frac{t}{2 \pi n}+\frac{1}{4}\right)^{-1 / 4}(g(t, n))^{-1}, \\
& g(t, n):=\operatorname{arsinh}\left(\left(\frac{\pi n}{2 t}\right)^{1 / 2}\right), \\
& f(t, n):=2 t g(t, n)+\left(2 \pi n t+\pi^{2} n^{2}\right)^{1 / 2}-\pi / 4,
\end{aligned}
$$$$
\text { where } 0<A<A^{\prime} \text { are any fixed constants. }
$$ 
Proof. This is the famous Atkinson formula (see Atkinson [1] or Ivić [5, Theorem 15.1]).

Lemma 8. Suppose $Y>1$. Define

$$
\begin{aligned}
c_{2}^{*} & :=\sum_{\sqrt{n}+\sqrt{m}=\sqrt{k}+\sqrt{l}} \frac{(-1)^{n+m+k+l} d(n) d(m) d(k) d(l)}{(n m k l)^{3 / 4}}, \\
c_{2}^{*}(Y) & :=\sum_{\substack{\sqrt{n}+\sqrt{m}=\sqrt{k}+\sqrt{l} \\
n, m, k, l \leq Y}} \frac{(-1)^{n+m+k+l} d(n) d(m) d(k) d(l)}{(n m k l)^{3 / 4}}, \\
c_{2}(Y) & :=\sum_{\substack{\sqrt{n}+\sqrt{m}=\sqrt{k}+\sqrt{l} \\
n, m, k, l \leq Y}} \frac{d(n) d(m) d(k) d(l)}{(n m k l)^{3 / 4}} .
\end{aligned}
$$

Then

$$
c_{2}=c_{2}^{*}, \quad c_{2}(Y)=c_{2}^{*}(Y), \quad\left|c_{2}-c_{2}(Y)\right| \ll Y^{-1 / 2+\varepsilon} .
$$

Proof. The estimate $\left|c_{2}-c_{2}(Y)\right| \ll Y^{-1 / 2+\varepsilon}$ is a special case of Lemma 3.1 of [13]. The equalities $c_{2}=c_{2}^{*}$ and $c_{2}(Y)=c_{2}^{*}(Y)$ follow from the fact that if $\sqrt{n_{1}}+\sqrt{n_{2}}=\sqrt{n_{3}}+\sqrt{n_{4}}$, then $n_{1}+n_{2}+n_{3}+n_{4}$ must be an even number.

LEMMA 9. If $Y>1$, then

$$
H_{1}(Y):=\sum_{\substack{\sqrt{n}+\sqrt{m}=\sqrt{k}+\sqrt{l} \\ n, m, k, l \leq Y}} \frac{d(n) d(m) d(k) d(l) \max (n, m, k, l)^{3}}{(n m k l)^{3 / 4}} \ll Y^{5 / 2+\varepsilon} .
$$

Proof. If $\sqrt{n}+\sqrt{m}=\sqrt{k}+\sqrt{l}$, then either

(1) $n=k, m=l$ or $n=l, m=k$, or

(2) $n \neq k, l$.

If (2) holds, then by a classical result of Besicovitch, we know that $n=n_{1}^{2} h, \quad m=m_{1}^{2} h, \quad k=k_{1}^{2} h, \quad l=l_{1}^{2} h, \quad n_{1}+m_{1}=k_{1}+l_{1}, \quad \mu(h) \neq 0$. Thus we get

$$
\begin{aligned}
H_{1}(Y) & \ll \Sigma_{1}+\Sigma_{2}, \\
\Sigma_{1} & \ll \sum_{n, k \leq Y} \frac{d^{2}(n) d^{2}(m) \max (n, k)^{3}}{(n k)^{3 / 2}} \ll Y^{5 / 2} \log ^{3} Y, \\
\Sigma_{2} & \ll Y^{\varepsilon} \sum_{h<Y} \sum_{\substack{n_{1}+m_{1}=k_{1}+l_{1} \\
n_{1}, m_{1}, k_{1}, l_{1} \leq Y^{1 / 2} h^{-1 / 2}}} \frac{\max \left(n_{1}, m_{1}, k_{1}, l_{1}\right)^{6}}{\left(n_{1} m_{1} k_{1} l_{1}\right)^{3 / 2}}
\end{aligned}
$$




$$
\begin{aligned}
& \ll Y^{\varepsilon} \sum_{h<Y} \sum_{\substack{n_{1}+m_{1}=k_{1}+l_{1} \\
n_{1}, m_{1}, l_{1} \leq k_{1} \leq Y^{1 / 2} h^{-1 / 2}}} \frac{k_{1}^{9 / 2}}{\left(n_{1} m_{1} l_{1}\right)^{3 / 2}} \sum_{\sum_{h<Y} \sum_{l_{1}} l_{1}^{-3 / 2} \sum_{\substack{n_{1}+m_{1}>k_{1} \\
n_{1}, m_{1} \leq k_{1} \leq Y^{1 / 2} h^{-1 / 2}}} \frac{k_{1}^{9 / 2}}{\left(n_{1} m_{1}\right)^{3 / 2}}} \sum_{\sum_{h<Y}^{\varepsilon} \sum_{l_{1}} l_{n_{1}}^{-3 / 2} \sum_{1}^{-3 / 2} k_{1}^{3}} \sum_{k_{1} \ll m_{1} \leq k_{1} \leq Y^{1 / 2} h^{-1 / 2}}\left(Y^{1 / 2} h^{-1 / 2}\right)^{5} \ll Y^{5 / 2+\varepsilon} .
\end{aligned}
$$

LEMMA 10. If $Y>1$, then

$$
H_{2}(Y):=\sum_{\substack{n+\sqrt{m}+\sqrt{k}=\sqrt{l} \\ n, m, k, l \leq Y}} \frac{d(n) d(m) d(k) d(l) l^{3 / 4}}{(n m k)^{3 / 4}} \ll Y^{1 / 2+\varepsilon} .
$$

Proof. If $\sqrt{n}+\sqrt{m}+\sqrt{k}=\sqrt{l}$, then

$n=n_{1}^{2} h, \quad m=m_{1}^{2} h, \quad k=k_{1}^{2} h, \quad l=l_{1}^{2} h, \quad n_{1}+m_{1}+k_{1}=l_{1}, \quad \mu(h) \neq 0$.

Thus we get

$$
\begin{aligned}
H_{2}(Y) & \ll Y^{\varepsilon} \sum_{h\left(n_{1}+m_{1}+k_{1}\right)^{2} \leq Y} \frac{\left(n_{1}+m_{1}+k_{1}\right)^{3 / 2}}{h^{3 / 2}\left(n_{1} m_{1} k_{1}\right)^{3 / 2}} \\
& \ll Y^{\varepsilon} \sum_{h<Y} h^{-3 / 2} \sum_{n_{1} \leq m_{1} \leq k_{1} \leq Y^{1 / 2} h^{-1 / 2}} n_{1}^{-3 / 2} m_{1}^{-3 / 2} \ll Y^{1 / 2+\varepsilon} .
\end{aligned}
$$

LEMMA 11. Suppose $f_{j}(t)(1 \leq j \leq k)$ and $g(t)$ are continuous, monotonic real-valued functions on $[a, b]$ and let $g(t)$ have a continuous, monotonic derivative on $[a, b]$. If $\left|f_{j}(t)\right| \leq A_{j}(1 \leq j \leq k),\left|g^{\prime}(t)\right| \gg \Delta$ for any $t \in[a, b]$, then

$$
\int_{a}^{b} f_{1}(t) \cdots f_{k}(t) e(g(t)) d t \ll A_{1} \cdots A_{k} \Delta^{-1} .
$$

Proof. This is Lemma 15.3 of Ivić [5].

5. Proof of Theorem 2. Suppose $T \geq 10$. It suffices to evaluate $\int_{T}^{2 T} E^{4}(t) d t$. Let $y:=T^{1 / 3-\varepsilon}$. For any $T \leq t \leq 2 T$, define

$$
\mathcal{E}_{1}(t):=\frac{1}{\sqrt{2}} \sum_{n \leq y} h(t, n) \cos (f(t, n)), \quad \mathcal{E}_{2}(t):=E(t)-\mathcal{E}_{1}(t) .
$$


From the inequality $(a+b)^{4}-a^{4} \ll|b|^{3}|a|+|b|^{4}$, we get

$$
\begin{aligned}
& \int_{T}^{2 T} E^{4}(t) d t \\
& \quad=\int_{T}^{2 T} \mathcal{E}_{1}^{4}(t) d t+O\left(\int_{T}^{2 T}\left|\mathcal{E}_{1}(t)\right|^{3}\left|\mathcal{E}_{2}(t)\right| d t\right)+O\left(\int_{T}^{2 T}\left|\mathcal{E}_{2}(t)\right|^{4} d t\right) .
\end{aligned}
$$

5.1. Evaluation of $\int_{T}^{2 T} \mathcal{E}_{1}^{4}(t) d t$. In this subsection, we shall evaluate the integral $\int_{T}^{2 T} \mathcal{E}_{1}^{4}(t) d t$. Similarly to Tsang [11], we can write

$$
\mathcal{E}_{1}^{4}(t)=\frac{3}{32} S_{5}(t)+\frac{3}{32} S_{6}(t)+\frac{1}{8} S_{7}(t)+\frac{1}{8} S_{8}(t)+\frac{1}{32} S_{9}(t),
$$

where

$$
\begin{aligned}
& S_{5}(t):=\sum_{\substack{n, m, k, l \leq y \\
\sqrt{n}+\sqrt{m}=\sqrt{k}+\sqrt{l}}} H(t ; n, m, k, l) \cos \left(F_{1}(t ; n, m, k, l)\right), \\
& S_{6}(t):=\sum_{\substack{n, m, k, l \leq y \\
\sqrt{n}+\sqrt{m} \neq \sqrt{k}+\sqrt{l}}} H(t ; n, m, k, l) \cos \left(F_{1}(t ; n, m, k, l)\right), \\
& S_{7}(t):=\sum_{\substack{n, m, k, l \leq y \\
\sqrt{n}+\sqrt{m}+\sqrt{k}=\sqrt{l}}} H(t ; n, m, k, l) \cos \left(F_{2}(t ; n, m, k, l)\right), \\
& \sum_{8}(t):=\sum_{\substack{n, m, k, l \leq y \\
\sqrt{n}+\sqrt{m}+\sqrt{k} \neq \sqrt{l}}} H(t ; n, m, k, l) \cos \left(F_{2}(t ; n, m, k, l)\right), \\
& \sum_{9}(t):=\quad H(t ; n, m, k, l) \cos \left(F_{3}(t ; n, m, k, l)\right), \\
& H(t ; n, m, k, l):=h(t, n) h(t, m) h(t, k) h(t, l), \\
& F_{1}(t ; n, m, k, l):=f(t, n)+f(t, m)-f(t, k)-f(t, l), \\
& F_{2}(t ; n, m, k, l):=f(t, n)+f(t, m)+f(t, k)-f(t, l), \\
& F_{3}(t ; n, m, k, l):=f(t, n)+f(t, m)+f(t, k)+f(t, l) .
\end{aligned}
$$
that

We first estimate the integral $\int_{T}^{2 T} S_{5}(t) d t$. For $n \leq y$, it is easy to check

$$
\begin{aligned}
h(t, n) & =\frac{2^{3 / 4}}{\pi^{1 / 4}} \frac{(-1)^{n} d(n)}{n^{3 / 4}} t^{1 / 4}\left(1+O\left(\frac{n}{t}\right)\right), \\
f(t, n) & =2^{3 / 2}(\pi n t)^{1 / 2}-\pi / 4+O\left(n^{3 / 2} t^{-1 / 2}\right), \\
f^{\prime}(t, n) & =2^{1 / 2}(\pi n)^{1 / 2} t^{-1 / 2}+O\left(n^{3 / 2} t^{-3 / 2}\right) .
\end{aligned}
$$


If $\sqrt{n}+\sqrt{m}=\sqrt{k}+\sqrt{l}$, then

$$
\cos \left(F_{1}(n, m, k, l)\right)=\cos \left(O\left(\frac{D^{3 / 2}}{t^{1 / 2}}\right)\right)=1+O\left(\frac{D^{3}}{t}\right),
$$

where $D:=\max (n, m, k, l)$. So from $(5.3),(5.6)$, and Lemmas 8 and 9 we get

$$
\begin{aligned}
& \int_{T}^{2 T} S_{5}(t) d t \\
= & \sum_{\substack{n, m, k, l \leq y \\
\sqrt{n}+\sqrt{m}=\sqrt{k}+\sqrt{l}}} \int_{T}^{2 T} H(t ; n, m, k, l) \cos \left(F_{1}(t ; n, m, k, l)\right) d t
\end{aligned}
$$$$
=\frac{8}{\pi} \sum_{\substack{n, m, k, l \leq y \\ \sqrt{n}+\sqrt{m}=\sqrt{k}+\sqrt{l}}} \frac{(-1)^{n+m+k+l} d(n) d(m) d(k) d(l)}{(n m k l)^{3 / 4}}
$$

$$
\begin{aligned}
& \times \int_{T}^{2 T} t\left(1+O\left(\frac{D}{t}\right)\right)\left(1+\left(\frac{D^{3}}{t}\right)\right) d t \\
& =\frac{8}{\pi} \quad \sum_{n, m, k, l \leq y} \frac{(-1)^{n+m+k+l} d(n) d(m) d(k) d(l)}{(n m k l)^{3 / 4}} \\
& \sqrt{n}+\sqrt{m}=\sqrt{k}+\sqrt{l} \\
& \times \int_{T}^{2 T} t\left(1+\left(\frac{D^{3}}{t}\right)\right) d t \\
& =\frac{8}{\pi} \sum_{\substack{n, m, k, l \leq y \\
\sqrt{n}+\sqrt{m}=\sqrt{k}+\sqrt{l}}} \frac{(-1)^{n+m+k+l} d(n) d(m) d(k) d(l)}{(n m k l)^{3 / 4}} \int_{T}^{2 T} t d t+O\left(T H_{1}(y)\right) \\
& =\frac{8 c_{2}}{\pi} \int_{T}^{2 T} t d t+O\left(T^{1+\varepsilon} y^{5 / 2}+T^{2+\varepsilon} y^{-1 / 2}\right) \\
& =\frac{8 c_{2}}{\pi} \int_{T}^{2 T} t d t+O\left(T^{11 / 6+\varepsilon}\right) .
\end{aligned}
$$

Now we estimate $\int_{T}^{2 T} S_{6}(t) d t$. From (5.5) we get

$$
F_{1}^{\prime}(t ; n, m, k, l)=(2 \pi)^{1 / 2} \eta t^{-1 / 2}+O\left(D^{3 / 2} t^{-3 / 2}\right),
$$

where $\eta=\sqrt{n}+\sqrt{m}-\sqrt{k}-\sqrt{l}$. Write 


$$
\int_{T}^{2 T} S_{6}(t) d t=\int_{|\eta| \leq T^{-1 / 2}} S_{6}(t) d t+\int_{|\eta|>T^{-1 / 2}} S_{6}(t) d t .
$$

If $|\eta| \leq T^{-1 / 2}$, then by (5.3) and the trivial estimate we get

$$
\int_{|\eta| \leq T^{-1 / 2}} S_{6}(t) d t \ll T^{2} \sum_{\substack{n, m, k, l \leq y ;|\eta| \leq T^{-1 / 2} \\ \sqrt{n}+\sqrt{m} \neq \sqrt{k}+\sqrt{l}}} \frac{d(n) d(m) d(k) d(l)}{(n m k l)^{3 / 4}} .
$$

If $|\eta|>T^{-1 / 2}$, then $\left|F_{1}^{\prime}(t ; n, m, k, l)\right| \gg|\eta| T^{-1 / 2}$, thus from (5.3) and Lemma 11 we get

$$
\int_{|\eta|>T^{-1 / 2}} S_{6}(t) d t \ll T^{3 / 2} \sum_{\substack{n, m, k, l \leq y ;|\eta|>T^{-1 / 2} \\ \sqrt{n}+\sqrt{m} \neq \sqrt{k}+\sqrt{l}}} \frac{d(n) d(m) d(k) d(l)}{(n m k l)^{3 / 4}|\eta|} .
$$

From (5.9), (5.10) and the estimate in Section 3 we get

$$
\begin{aligned}
\int_{T}^{2 T} S_{6}(t) d t & \ll \sum_{\substack{n, m, k, l \leq y \\
\sqrt{n}+\sqrt{m} \neq \sqrt{k}+\sqrt{l}}} \frac{d(n) d(m) d(k) d(l)}{(n m k l)^{3 / 4}} \min \left(T^{2}, T^{3 / 2}|\eta|^{-1}\right) \\
& \ll T^{53 / 28+\varepsilon} .
\end{aligned}
$$

If $\sqrt{n}+\sqrt{m}+\sqrt{k}=\sqrt{l}$, then from (5.4) we have

$F_{2}(t ; n, m, k, l)=-\pi / 2+O\left(l^{3 / 2} t^{-1 / 2}\right), \quad \cos \left(F_{2}(t ; n, m, k, l)\right) \ll l^{3 / 2} t^{-1 / 2}$.

Thus from (5.3), the trivial estimate and Lemma 10 we get

$$
\int_{T}^{2 T} S_{7}(t) d t \ll T^{3 / 2} H_{2}(y) \ll T^{3 / 2} y^{1 / 2+\varepsilon} \ll T^{5 / 3+\varepsilon} .
$$

Similarly to the integral $\int_{T}^{2 T} S_{6}(t) d t$, we have

$$
\int_{T}^{2 T} S_{8}(t) d t \ll T^{53 / 28+\varepsilon} \text {. }
$$

From (5.5) we get

$$
F_{3}^{\prime}(t ; n, m, k, l) \gg(\sqrt{n}+\sqrt{m}+\sqrt{k}+\sqrt{l}) T^{-1 / 2},
$$

which together with (5.3) and Lemma 11 implies

$$
\begin{aligned}
& \int_{T}^{2 T} S_{9}(t) d t \\
& \ll \sum_{n, m, k, l} \frac{d(n) d(m) d(k) d(l) T^{3 / 2}}{(n m k l)^{3 / 4}(\sqrt{n}+\sqrt{m}+\sqrt{k}+\sqrt{l})} \ll T^{3 / 2+\varepsilon} y^{1 / 2} \ll T^{5 / 3+\varepsilon} .
\end{aligned}
$$


From (5.2), (5.7), (5.11)-(5.14) we get

$$
\int_{T}^{2 T} \mathcal{E}_{1}^{4}(t) d t=\frac{3 c_{2}}{4 \pi} \int_{T}^{2 T} t d t+O\left(T^{53 / 28+\varepsilon}\right) .
$$

5.2. Completion of proof of Theorem 2. Let $A_{0}=35 / 8$. Ivić [5, Thm. 15.7] proved the estimate

$$
\int_{1}^{T}|E(t)|^{A_{0}} d t \ll T^{1+A_{0} / 4+\varepsilon} .
$$

By his method we can show

$$
\int_{T}^{2 T}\left|\mathcal{E}_{1}(t)\right|^{A_{0}} d t \ll T^{1+A_{0} / 4+\varepsilon} .
$$

Thus

$$
\int_{T}^{2 T}\left|\mathcal{E}_{2}(t)\right|^{A_{0}} d t \ll T^{1+A_{0} / 4+\varepsilon}
$$

We also have

$$
\int_{T}^{2 T}\left|\mathcal{E}_{2}(t)\right|^{2} d t \ll T^{3 / 2+\varepsilon} y^{-1 / 2},
$$

which is formula (4.15) of [14]. From (5.18), (5.19) and Hölder's inequality the estimate

$$
\int_{T}^{2 T}\left|\mathcal{E}_{2}(t)\right|^{A} d t \ll T^{1+A / 4+\varepsilon} y^{-\left(A_{0}-A\right) / 2\left(A_{0}-2\right)}
$$

holds for any $2<A<A_{0}$. The details can be found in [14].

From (5.17), (5.20) and Hölder's inequality we get

$$
\begin{aligned}
\int_{T}^{2 T}\left|\mathcal{E}_{1}^{3}(t) \mathcal{E}_{2}(t)\right| d t & \ll\left(\int_{1}^{T}\left|\mathcal{E}_{1}(t)\right|^{A_{0}} d t\right)^{3 / A_{0}}\left(\int_{1}^{T}\left|\mathcal{E}_{2}(t)\right|^{A_{0} /\left(A_{0}-3\right)} d t\right)^{\left(A_{0}-3\right) / A_{0}} \\
& \ll T^{2+\varepsilon} y^{-\left(A_{0}-4\right) / 2\left(A_{0}-2\right)} \ll T^{2-19 / 108+\varepsilon}
\end{aligned}
$$

From (5.1), (5.15), (5.20) with $A=4$ and (5.21) we get

$$
\int_{T}^{2 T} E^{4}(t) d t=\frac{3 c_{2}}{4 \pi} \int_{T}^{2 T} t d t+O\left(T^{53 / 28+\varepsilon}\right)
$$

and Theorem 2 follows. 


\section{References}

[1] F. V. Atkinson, The mean-value of the Riemann zeta-function, Acta Math. 81 (1949), 353-376.

[2] Y. C. Cai, On the third and fourth power moments of Fourier coefficients of cusp forms, Acta Math. Sinica (N.S.) 13 (1997), 443-452.

[3] M. N. Huxley, Exponential sums and lattice points III, Proc. London Math. Soc. (3) 87 (2003), 591-609.

[4] A. Ivić, On some problems involving the mean square of $\zeta(1 / 2+i t)$, Bull. Cl. Sci. Math. Nat. Sci. Math. 23 (1998), 71-76.

[5] - The Riemann Zeta-Function, Wiley, 1985.

[6] - Lectures on Mean Values of the Riemann Zeta-Function, Tata Inst. Fund. Res. Lectures on Math. and Phys. 82, Bombay, 1991.

[7] A. Ivić and P. Sargos, On the higher power moments of the error term in the divisor problem, to appear.

[8] M. Jutila, On a formula of Atkinson, in: Topics in Classical Number Theory (Budapest, 1981), Vols. I, II, Colloq. Math. Soc. János Bolyai 34, North-Holland, Amsterdam, 1984, 807-823.

[9] O. Robert and P. Sargos, Three-dimensional exponential sums with monomials, J. Reine Angew. Math., in print.

[10] K. C. Tong, On divisor problem III, Acta Math. Sinica 6 (1956), 515-541.

[11] K. M. Tsang, Higher-power moments of $\Delta(x), E(t)$ and $P(x)$, Proc. London Math. Soc. (3) 65 (1992), 65-84.

[12] W. G. Zhai, On higher-power moments of $\Delta(x)$, Acta Arith. 112 (2004), 367-395.

[13] -, On higher-power moments of $\Delta(x)$ (II), ibid. 114 (2004), 35-54.

[14] -, On higher-power moments of E(t), ibid. 115 (2004), 329-348.

Department of Mathematics

Shandong Normal University

Jinan 250014, P.R. China

E-mail: zhaiwg@hotmail.com

Received on 20.5.2004

and in revised form on 24.12.2004 